\title{
ORIGINAL
}

\section{IMPACTO DE LOS INGRESOS URGENTES INNECESARIOS SOBRE LAS ESTANCIAS HOSPITALARIAS EN UN HOSPITAL DE ASTURIAS}

\author{
Luis Velasco Díaz (1), Susana García Ríos (1), David Oterino de la Fuente (2,3), Francisco Suárez \\ García (4), Susana Diego Roza (1) y Reyes Fernández Alonso (1) \\ (1) Hospital Valle del Nalón. \\ (2) Fundación Instituto de Investigación en Servicios de Salud. \\ (3) Centro Salud Teatinos. Oviedo. \\ (4) Consejería de Salud y Servicios Sanitarios del Principado de Asturias.
}

\section{RESUMEN}

Fundamento: Los ingresos innecesarios en los hospitales de agudos tienen importantes consecuencias sobre su eficiencia y organización. El objetivo de este estudio es identificar el grado de inadecuación de los ingresos realizados desde un servicio de urgencias hospitalario y sus causas, así como cuantificar las estancias inadecuadas generadas por estos ingresos.

Método: Se evaluó la adecuación de 622 ingresos realizados en al año 2002 seleccionados aleatoriamente, y la del total de estancias generadas por los ingresos inadecuados y una muestra representativa de los ingresos adecuados de un hospital de segundo nivel de Asturias. El instrumento de revisión fue el Appropiateness Evaluation Protocol. Se realizó un análisis descriptivo de la muestra, un análisis bivariante y un análisis de regresión logística multivariante.

Resultados: Se consideraron inadecuados 63 ingresos $(10,1 \%)$ La principal causa de inadecuación fueron los ingresos para realizar pruebas diagnósticas y/o tratamientos que podrían realizarse de forma ambulatoria. Los ingresos innecesarios generaron un $78,2 \%$ de estancias innecesarias y los necesarios un $24,8 \%$. Incrementaron el riesgo de ingresos innecesarios la derivación a urgencias desde consultas externas del propio hospital (OR:4,50, IC 95\%: 1,59-12,76), ingresar en horario de mañana (OR: 13,97, IC 95\%: 1,86-104,76) o tarde (OR: 7,70, IC 95\%: 1,01-58,72), ingresar en los servicios de cardiología (OR: 3,93, IC 95\%: 1,22-12,70) y neurología (OR: 5,86, IC 95\%: 1,88-18,30) disminuyó el riesgo de ingreso innecesario la experiencia de ingresos previos (OR: 0,34, IC 95\%: 0,18-0,65).

Conclusiones: Los ingresos innecesarios generan tres veces más estancias inadecuadas que los necesarios. Los problemas organizativos del centro son la principal causa de ingresos inadecuados.

Palabras clave: Alta del Paciente. Hospitales. Servicio de Admisión en Hospital

Correspondencia:

Dr. Luis Velasco Díaz

Servicio de Urgencias Hospital Valle del Nalón.

Riaño

Asturias

Correo electrónico: luykys2@telefonica.net
ABSTRACT

\section{Impact on Hospital Days of Care Due to Unnecessary Emergency Admissions}

Background: Unnecessary admissions of acute cases have major impacts on hospital efficiency and organization. This study is aimed to identify percentage of unnecessary admissions from a hospital emergency department and the reasons why, as well as to quantify the unnecessary hospital days of care generated by these admissions.

Methods: It has been analyzed the appropriateness of 622 admissions made in 2002, selected at random, all of the hospital stays generated by the inappropriate admissions and a representative sample of the appropriate admissions of a second-level hospital in Asturias. The review tool was the Appropriateness Evaluation Protocol. A descriptive analysis, a bivariate analysis and a multivariate logic regression analysis were made.

Results: A total of 63 admissions $(10.1 \%)$ were judged inappropriate. The main cause of inappropriateness were admissions for performing diagnostic tests and/or treatments, which could be carried out on an outpatient basis. These unnecessary admissions generated $78.2 \%$ of unnecessary stays, and the appropriate admissions generated $24.8 \%$ of unnecessary stays. Referrals to hospital emergency rooms from physicians outside of the hospital proper heightened the risk of unnecessary admissions (OR:4.50, 95\% CI: 1.59-12.76), daytime admissions (OR: 13.97, 95\% CI: 1.86-104.76) or evening admissions (OR: $7.70,95 \%$ CI: 1.01-58.72), admissions to cardiology wards (OR: 3.93, 95\% CI: $1.22-12.70)$ and neurology wards (OR: $5.86,95 \% \mathrm{CI}$ : 1.88-18.30), the experience of prior admissions having lowered the risk of unnecessary admission (OR: $0.34,95 \%$ CI: 0.18-0.65)

Conclusions: Unnecessary admissions generate three times more inappropriate stays than the necessary admissions. Hospital organization-related problems are the main cause of inappropriate admissions.

Key words: Admitting Department, Hospital. Patient Discharge. Hospitals. 


\section{INTRODUCCIÓN}

Los ingresos inadecuados en hospitales de agudos sigue siendo tema de interés después de dos décadas de revisión con instrumentos basados en criterios explícitos ${ }^{1-8}$. Las hospitalizaciones inadecuadas en servicios médicos se mantienen entre el 10 y $20 \%$, aunque algunos estudios han observado una disminución ${ }^{9}$. Las principales causas de inadecuación ${ }^{4,5,10-13}$ (ingresos para realizar procedimientos diagnósticos y/o terapéuticos que podrían realizarse ambulatoriamente, problemas de organización del hospital y el estilo de practica de los médicos) tampoco varían, y mientras que algunas intervenciones como el AdeQuos ${ }^{14}$ o unidades de corta estancia han mostrado su efectividad ${ }^{8}$, otras como la retroinformación a los clínicos ofrecen resultados variables ${ }^{15-17}$.

El incremento de los costes sanitarios es uno de los argumentos principales en el desarrollo de la revisión de la utilización de los recursos para disminuir la hospitalización inadecuada. Es necesario tener en cuenta que el posible ahorro derivado de la evitación de estancias inadecuadas, tiene distinto significado para los hospitales, los usuarios y los pagadores, y su mayor o menor beneficio (para el hospital) puede estar relacionado con el índice de ocupación, la necesidad de concertar camas, el sistema de financiación y una buena gestión para utilizar adecuadamente las camas «liberadas». Por esto Meneu ${ }^{18}$ propone diversos «beneficios no meramente contables» de la revisión del uso inadecuado, como la identificación de la hospitalización inadecuada y sus causas para definir intervenciones vinculadas a objetivos de gestión, disponer de información relevante para la planificación sanitaria con criterios objetivos, o valorar la responsabilidad de los distintos estamentos en hospitalización inadecuada.

La relevancia de los ingresos inadecuados se deriva del mayor número de estancias que originan $^{10,16,20}$. El objetivo de este trabajo es identificar el grado de inadecuación de los ingresos realizados desde el Servicio de Urgencias Hospitalario (SUH) en servicios médicos y sus causas y cuantificar las estancias inadecuadas generadas por estos ingresos.

\section{MATERIAL Y MÉTODOS}

Se revisaron las historias clínicas de las personas ingresadas en servicios médicos desde el SUH en el año 2002, para valorar la adecuación del ingreso y de las estancias generadas en el hospital Valle del Nalón, centro de referencia del área sanitaria de Langreo (Asturias). Se trata de un hospital comarcal de segundo nivel que dispone de 251 camas (106 asignadas a los servicios médicos) para atender a 83.302 personas. En el año 2002 ingresó un total de 2.874 pacientes, de los que 149 correspondieron a ingresos programados. Se atendió un total de 27.193 urgencias, de las cuales ingresaron $4.672(17,2 \%)$.

La muestra $(n=622)$, estimada para cada uno de los servicios médicos con una precisión del 6\%, un error alfa del $5 \%$ y una prevalencia de ingresos inadecuados del $15 \%$, se estratificó por grupos de edad (15-44, 4564, 65-74 y 75 y más), y se aumentó en un $10 \%$ para compensar posibles pérdidas. En los servicios de nefrología y endocrinología, con 28 y 35 altas respectivamente, se revisaron todas las altas. La selección de las historias se realizó por muestreo aleatorio simple desde la base de datos de admisiones del hospital. El instrumento de revisión fue la versión validada para España del Appropiateness Evaluation Proticol ${ }^{19}$ (AEP). Los revisores, cuatro médicos participantes en el estudio, que alcanzaron un acuerdo interobservador superior al $85 \%$ con un revisor experto en el uso del AEP, revisaron las historias clínicas para identificar la adecuación del ingreso. En cuanto a las estancias se revisó el total de las generadas por los ingresos inadecuados y uno de cada tres de los ingre- 
sos adecuados $(\mathrm{n}=184)$ seleccionados sistemáticamente.

La variable de estudio fue la adecuación del ingreso (si/no) y las variables independientes eran la adecuación de la estancia (si/no), los motivos de la inadecuación de los ingresos y estancias según el listado del AEP, edad, sexo, servicio de alta, (medicina interna, neumología, cardiología, neurología, digestivo, endocrino/nefrología), distancia desde la zona de salud de procedencia al hospital, orden de asistencia (espontáneo, atención primaria, consultas externas hospitalarias), estación del año, día de la semana, hora de llegada a urgencias (mañana: 6,0114,00, tarde: 14,01-22,00, noche: 22,01$6,00)$, patologías crónicas asociadas, diagnóstico principal que motivó el ingreso (Clasificación Internacional de Enfermedades CIE 9-Modificación Clínica), pruebas diagnósticas realizadas en urgencias (hemograma, bioquímica sangre/orina, coagulación sanguínea, gasometría, radiología, TAC, ecografía, ECG, cultivo), ingresos hospitalarios y visitas a urgencias en el ultimo año.

Análisis Estadístico: Se realizó un análisis univariante para describir la muestra (media e intervalo de confianza al $95 \%$ para las variables cuantitativas y frecuencias para las categóricas) y análisis bivariante con las variables de interés para establecer relaciones, utilizándose el estadístico chi cuadrado. Para controlar la confusión e interacción con las variables que alcanzaron significación estadística en el análisis bivariante se realizó un análisis de regresión logística multivariante (variable dependiente ingreso adecuado si/no), por el método stepwise, con un valor de p menor de 0,05 para la inclusión y menor 0,10 para su exclusión. Los análisis se realizaron con el SPSS 11.0.

\section{RESULTADOS}

La edad media de las personas ingresadas fue de 69,2 años, (IC 95\%:68,2-70,7) siendo el 45,8\% mayores de 74 años. Acudieron espontáneamente a urgencias el $64,7 \%$ y el $15,9 \%$ en horario nocturno, el $83,8 \%$ tenía dos o más patologías crónicas, casi la mitad de los pacientes $(48,9 \%)$ acudieron a urgencias por enfermedades del aparato circulatorio y respiratorio; al 75,9\% se le realizó más de cinco pruebas diagnósticas en urgencias. El 46,6\% había tenido uno o más ingresos hospitalarios, el $65,5 \%$ había acudido al SUH al menos una vez el año anterior y la estancia media fue de 11,0 días (IC95\%:10,3-11,7 (tabla 1).

De los 622 ingresos revisados $63(10,1 \%)$ se consideraron inadecuados, el porcentaje de inadecuación fue significativamente más elevado en los pacientes que procedían de consultas externas del propio hospital $(30,4 \%)$, los que tenían una o ninguna patología crónica $(18,0 \%)$, los que no tenían ninguna visita al SUH $(15,8 \%)$, ni ingreso hospitalario en el año anterior $(14,7 \%)$, los ingresados en el servicio de neurología $(21,4 \%)$, los que acuden a urgencias entre las 6 y 14 horas (14,9\%), y los pacientes dados de alta por tumores $(36,4 \%$ ) (tabla 1$)$. La mayor parte de la inadecuación de los ingresos hospitalarios $(73,0 \%)$ fue debida a ingresos para realizar pruebas diagnósticas y/o tratamientos que podrían haberse realizado de forma ambulatoria (tabla 2).

El análisis de regresión logística de predicción de ingresos inadecuados reveló que la procedencia de las consultas externas del propio hospital, el acudir al SUH en el horario de mañana y tarde e ingresar en los servicios de cardiología y neurología aumentan el riesgo de ingresos inadecuados. Por el contrario, haber ingresado el año anterior disminuiría el riesgo de ingreso inadecuado. (tabla 3).

En los ingresos innecesarios el porcentaje de estancias inadecuadas fue del $78,0 \%$ (571), mientras que la muestra de ingresos necesarios -sin diferencias significativas respecto al total de ingresos adecuados- 
Tabla 1

Características de los ingresos y porcentaje de inadecuación

\begin{tabular}{|c|c|c|c|c|c|}
\hline & & \multirow{2}{*}{$\begin{array}{c}\begin{array}{c}\text { Total } \\
\text { ingresos }\end{array} \\
\mathbf{n}\end{array}$} & \multicolumn{3}{|c|}{ Ingresos inadecuados } \\
\hline & & & $\%$ & $\mathbf{n}$ & $\%$ \\
\hline \multirow[t]{2}{*}{ Sexo } & Hombre & 361 & $58 \%$ & 35 & 9,7 \\
\hline & Mujer & 261 & $42 \%$ & 28 & 10,7 \\
\hline \multirow[t]{3}{*}{ Edad } & $15-64$ & 169 & 27,2 & 21 & 12,4 \\
\hline & $65-74$ & 168 & 27 & 18 & 10,7 \\
\hline & $>74$ & 285 & 45,8 & 24 & 8,4 \\
\hline \multirow[t]{3}{*}{ Orden asistencia* } & Espontáneo & 427 & 68,8 & 39 & 9,1 \\
\hline & Atención Primaria & 171 & 27,5 & 17 & 9.9 \\
\hline & Consultas Externas & 23 & 3,7 & 7 & 30,4 \\
\hline \multirow[t]{3}{*}{ Hora de llegada* } & Mañana & 261 & 42.0 & 39 & 14,9 \\
\hline & Tarde & 262 & 42,1 & 23 & 8,8 \\
\hline & Noche & 99 & 15,9 & 1 & 1,0 \\
\hline \multirow[t]{7}{*}{ Día semana } & Lunes & 91 & 14,6 & 6 & 6,6 \\
\hline & Martes & 110 & 17,7 & 16 & 14,5 \\
\hline & Miércoles & 95 & 15,3 & 13 & 13,7 \\
\hline & Jueves & 89 & 14,3 & 8 & 9,0 \\
\hline & Viernes & 96 & 15,4 & 10 & 10,4 \\
\hline & Sábado & 70 & 11,3 & 5 & 7,1 \\
\hline & Domingo & 71 & 11,4 & 5 & 7,0 \\
\hline \multirow[t]{4}{*}{ Estación del año } & Invierno & 150 & 24,1 & 14 & 9,3 \\
\hline & Primavera & 142 & 22,8 & 11 & 7,7 \\
\hline & Verano & 158 & 25,4 & 17 & 10,8 \\
\hline & Otoño & 172 & 27,7 & 21 & 12,2 \\
\hline \multirow{10}{*}{$\begin{array}{l}\text { Diagnóstico al alta según CIE } \\
9 *\end{array}$} & I. Enf. infecciosas y parasitarias & 19 & 3,1 & 2 & 10,5 \\
\hline & II. Tumores & 22 & 3,5 & 8 & 36,4 \\
\hline & III. Enf. de las glándulas endocrinas, nutrición.... & 34 & 5,5 & 1 & 2,9 \\
\hline & IV. Enf. de la sangre y órganos hematopoyéticos & 17 & 2,7 & 4 & 23,5 \\
\hline & V. Trastornos mentales & 17 & 2,7 & 3 & 17,6 \\
\hline & VI. Enf. del sistema nervioso y órganos de los sentidos & 85 & 13,7 & 15 & 17,6 \\
\hline & VII. Enf. del aparato circulatorio & 156 & 25,1 & 11 & 7,1 \\
\hline & VIII. Enf. del aparato respiratorio & 148 & 23,8 & 1 & 0,7 \\
\hline & IX. Enf. del aparato digestivo & 85 & 13,7 & 4 & 4,7 \\
\hline & Otros & 39 & 6,3 & 14 & 35,9 \\
\hline \multirow[t]{4}{*}{ Distancia al hospital } & $0-5 \mathrm{Km}$ & 293 & 47,1 & 31 & 10,6 \\
\hline & $5,1-15 \mathrm{Km}$ & 179 & 28,8 & 18 & 10,1 \\
\hline & $>15 \mathrm{Km}$ & 107 & 17,2 & 8 & 7,5 \\
\hline & Fuera del área & 43 & 6,9 & 6 & 14 \\
\hline \multirow[t]{2}{*}{ Patologías crónicas* } & $0-1$ & 100 & 16,1 & 18 & 18 \\
\hline & 2 o más & 522 & 83,9 & 45 & 8,6 \\
\hline \multirow[t]{2}{*}{ Ingresos el ultimo año* } & Ninguno & 333 & 53,5 & 49 & 14,7 \\
\hline & Uno o más & 289 & 46,5 & 14 & 4,8 \\
\hline \multirow{2}{*}{$\begin{array}{l}\text { Visitas a urgencias ultimo } \\
\text { año* }\end{array}$} & Ninguna & 215 & 34,6 & 34 & 15,8 \\
\hline & Una o más & 407 & 65,4 & 29 & 7,1 \\
\hline \multirow[t]{2}{*}{ Pruebas diagnósticas* } & $0-4$ & 150 & 24.1 & 21 & 14 \\
\hline & Cinco o más & 472 & 75,9 & 42 & 8,9 \\
\hline \multirow[t]{3}{*}{ Estancias } & 1-3 estancias & 76 & 12,2 & 9 & 11,8 \\
\hline & 4-8 estancias & 210 & 33,8 & 18 & 8,6 \\
\hline & Mas de 8 estancias & 336 & 54,0 & 36 & 10,7 \\
\hline
\end{tabular}


Tabla 1 (Cont.)

\begin{tabular}{|l|l|r|r|r|r|}
\hline Servicio de alta* & Neumología & 127 & 20,4 & 4 & 3,1 \\
\cline { 2 - 5 } & Cardiología & 125 & 20,1 & 14 & 11,2 \\
\cline { 2 - 5 } & Medicina Interna & 114 & 18,3 & 12 & 10,5 \\
\cline { 2 - 5 } & Neurología & 103 & 16,6 & 22 & 21,4 \\
\cline { 2 - 5 } & Digestivo & 107 & 17,2 & 9 & 8,4 \\
\cline { 2 - 5 } & Endocrino/Nefrología & 46 & 7,4 & 2 & 4,4 \\
\hline
\end{tabular}

- $\mathrm{p}<0,05$

Tabla 2

Causas de admisión inadecuada

\begin{tabular}{|lcc|}
\hline & $\begin{array}{c}\text { Numero } \\
\text { ingresos }\end{array}$ & $\%$ \\
\hline Pruebas diagnósticas y/o tratamientos pueden realizarse en consultas externas & 46 & 73,0 \\
\hline $\begin{array}{l}\text { El paciente ha sido ingresado para realizar pruebas diagnósticas y/o tratamiento que pueden } \\
\text { realizarse en consultas externas, excepto si el paciente vive muy lejos como para efectuarse de } \\
\text { forma rápida }\end{array}$ & 9 & 14,3 \\
\hline Otras & 8 & 12,7 \\
\hline Total & 62 & 100 \\
\hline
\end{tabular}

Tabla 3

Inadecuación de los ingresos. Análisis de regresión logística

\begin{tabular}{|c|c|c|c|c|}
\hline \multirow{3}{*}{ Orden asistencia } & \multirow{2}{*}{ Odd Ratio } & \multicolumn{2}{|c|}{ Intervalo Confianza 95,0\% } & \multirow{2}{*}{ Significación } \\
\hline & & \multirow[t]{2}{*}{ Inferior } & \multirow{2}{*}{ Superior } & \\
\hline & & & & \\
\hline & 1,000 & & & \\
\hline Atención Primaria & 1,056 & 0,562 & 1,984 & NS $(0,865)$ \\
\hline Consultas externas & 4,503 & 1,589 & 12,755 & 0,005 \\
\hline \multicolumn{5}{|l|}{ Ingresos previos } \\
\hline Ninguno & 1,000 & & & \\
\hline Uno o más & 0,339 & 0,177 & 0,650 & 0,001 \\
\hline \multicolumn{5}{|l|}{ Servicio de ingreso } \\
\hline Neuomología & 1,000 & & & \\
\hline Medicina Interna & 3,119 & 0,949 & 10,252 & NS $(0,061)$ \\
\hline Cardiología & 3,934 & 1,219 & 12,698 & 0,022 \\
\hline Nefro/Endocrino & 0,802 & 0,134 & 4,804 & NS $(0,809)$ \\
\hline Neurología & 5,861 & 1,878 & 18,294 & 0,002 \\
\hline Digestivo & 2,636 & 0,765 & 9,084 & NS $(0,125)$ \\
\hline \multicolumn{5}{|l|}{ Hora de ingreso } \\
\hline Noche & 1,000 & & & \\
\hline Mañana & 13,968 & 1,862 & 104,760 & 0,010 \\
\hline Tarde & 7,698 & 1,009 & 58,719 & 0,049 \\
\hline
\end{tabular}


Tabla 4

Causas de las estancias inadecuadas según la adecuación del ingreso

\begin{tabular}{|l|c|c|c|c|c|c}
\hline \multirow{2}{*}{} & \multicolumn{2}{|c|}{ Ingreso adecuado } & \multicolumn{2}{c}{ Ingreso inadecuado } & \multicolumn{3}{c}{ Total } \\
\cline { 2 - 7 } & $\mathbf{n}$ & $\mathbf{\%}$ & $\mathbf{n}$ & $\mathbf{\%}$ & $\mathbf{n}$ & $\%$ \\
\hline $\begin{array}{l}\text { Retraso en el desarrollo del estudio o tratamiento para el que } \\
\text { el paciente está hospitalizado }\end{array}$ & 95 & 18,3 & 88 & 15,4 & 183 & 16,8 \\
\hline Responsabilidad del médico o del hospital & 404 & 77,7 & 476 & 83,4 & 880 & 80,7 \\
\hline Responsabilidad del médico o de la familia del paciente & 3 & 0,6 & 7 & 1,2 & 10 & 0,9 \\
\hline $\begin{array}{l}\text { Responsabilidad del medio (No disponibilidad de una } \\
\text { instalación alternativa) }\end{array}$ & 18 & 3,5 & & 0,0 & 18 & 1,6 \\
\hline Total & 520 & 100,0 & 571 & 100,0 & 1091 & 100,0 \\
\hline
\end{tabular}

generó un 24,8\% de estancias inadecuadas (520). Los problemas organizativos del servicio o del hospital fueron la causa de la mayoría de las estancias inadecuadas, sin diferencias significativas respecto a la necesidad del ingreso (tabla 4).

\section{DISCUSIÓN}

Los resultados obtenidos en este estudio ponen de manifiesto que los ingresos innecesarios tienen un fuerte impacto sobre las estancias hospitalarias, ya que generan tres veces más estancias inadecuadas que los ingresos necesarios. Se observa además que algunos factores, como ingresar en los servicios de cardiología y neurología, la procedencia de las consultas externas del propio hospital, la hora del ingreso y la experiencia previa de ingresos hospitalarios se asocian a la necesidad del ingreso.

En relación al diseño del estudio deben señalarse algunas limitaciones a tener en cuenta en la interpretación de los resultados obtenidos. Así, la calidad de la historia clínica puede repercutir tanto en la clasificación de un ingreso adecuado/inadecuado como en las posibles causas de inadecuación, ya que el AEP asume que los datos esenciales para determinar la necesidad de la hospitalización y sus causas se hallan en la historia clínica, el sistema de muestreo -revisión de todas las estancias de cada ingreso seleccionado- infraestima ligeramente la proporción de estancias inadecuadas, pero puede producir sesgos en las variables asociadas al uso inadecuado en relación a la duración de la estancia, (en los ingresos más prolongados se observa mayor proporción de estancias inadecuadas) por lo que hay que tenerlo en cuenta al valorar estos resultados ${ }^{20}$.

Por último, las limitaciones propias del AEP, ya que aunque ha demostrado su validez y una elevada fiabilidad interobservador ${ }^{19}$, no tiene en cuenta el uso inapropiado de procedimientos diagnósticos y terapéuticos, ya que siempre valora como adecuado el juicio clínico, considera disponibles los recursos alternativos a la hospitalización y no considera las expectativas de los enfermos y familiares ${ }^{3}$. Además el AEP detecta como innecesarias estancias de escasa intensidad diagnóstica y de consumo de recur$\operatorname{sos}^{18}$ lo que debe valorarse en su planificación.

La edad $^{12}$ y el diagnóstico ${ }^{5,21}$, que en otros estudios se asocian a la inadecuación del ingreso, no se asocian en éste, aunque hay otros estudios en los que tampoco se asocia la edad ${ }^{13,22}$ a la hospitalización inadecuada. Esta disparidad podría estar relacionada con la metodología del estudio (muestreo), los grupos de pacientes incluidos o las clasifica- 
ciones del diagnóstico de ingreso utiliza$\operatorname{das}^{12}$.

El incremento de riesgo de ingreso inadecuado cuando el paciente es derivado desde las consultas externas del propio hospital se ha observado en otros estudios ${ }^{12,23}$ pone en evidencia problemas de organización hospitalaria para la agilización de diagnósticos o procesos terapéuticos, auque la literatura en este aspecto es escasa.

Los ingresos hospitalarios previos y la comorbilidad (más de una patología crónica) podrían estar relacionados probablemente con la mayor intensidad del tratamiento de estos pacientes (detectada por el AEP) aunque en otros estudios estos factores no se asocian a la hospitalización inadecuada ${ }^{13}$.

Por su parte las diferencias entre servicios en los ingresos inadecuados ${ }^{1,6,24}$ podrían estar relacionadas con la especialidad medica y el tipo de pacientes que atienden, pero sobre todo con las características de los médicos, estilo de practica, pautas diagnósticas y de tratamiento, la organización interna, y su relación con el resto de los servicios hospitalarios $^{6}$.

Las causas más frecuentes de ingreso inadecuado son los tratamientos y/o las pruebas diagnósticas que podrían ser realizadas ambulatoriamente y, en lo referente a las estancias, casi la mitad de las inadecuadas se asocian a problemas organizativos del hospital o del servicio (retrasos en el desarrollo de exploraciones para las que el paciente fue ingresado y que son necesarias para tomar decisiones, o prolongación del ingreso por demora en la recepción de resultados de pruebas diagnósticas o interconsultas) lo que orienta a problemas de organización interna del hospital y de los servicios.

La relevancia de los ingresos inadecuados deriva de las estancias inadecuadas que originan. En la mayoría de los estudios se observa una asociación entre ingresos inade- cuados y estancias inadecuadas ${ }^{10,11,16}$. Por ello, las intervenciones para reducirlos podrían tener una fuerte repercusión sobre las estancias inadecuadas, ya que un pequeño número de ingresos $(10,1 \%)$, son los responsables de la mitad de las estancias inadecuadas $(52,43 \%)$.

En los últimos años se han desarrollado diversas estrategias circunscritas al ámbito hospitalario para mejorar la utilización de los hospitales, agilizando la realización de pruebas diagnósticas y tratamientos (hospitalización de día o domiciliaria, unidades de corta estancia, consultas de alta resolución, cirugía mayor ambulatoria), racionalización de la practica clínica (vías clínicas, guías de practica), incremento en la oferta de recursos socio-sanitarios (unidades de cuidados paliativos, hospitales de media y larga estancia), intervenciones sobre los clínicos para disminuir el uso innecesario (retroinformación de resultados, AdeQuos). Sin embargo, el problema de fondo parece continuar y es que la prioridad para realizar determinadas pruebas diagnósticas se establece en función de dónde está el paciente (sala de hospitalización general, unidad de alta resolución, consultas externas o atención primaria), y no de criterios clínicos ${ }^{25}$.

La capacidad de la atención primaria para disminuir los ingresos inadecuados es una estrategia no estudiada hasta la fecha, a pesar de que algunos estudios e informes han señalado su interes ${ }^{25-27}$, por ello, y considerando que la realización de pruebas diagnósticas y/o tratamientos que podrían realizarse de forma ambulatoria es la principal causa de inadecuación de los ingresos, la relevancia de una atención primaria fuerte 28-31 $^{28}$ (incremento de la capacidad resolutiva, coordinación entre primaria y especializada para mejorar la gestión del circuito de exploraciones complementarias e interconsultas), como una estrategia útil para disminuir la hospitalización inadecuada debería incluirse en los próximos estudios sobre inadecuación de ingresos y estancias hospitalarias. 


\section{BIBLIOGRAFÍA}

1. Payne SM. Identifying and managing inappropriate hospital utilization: a policy synthesis. Health Serv Res. 1987 Dec; 22(5):709-69.

2. Restucia JD. The evolution of hospital utilization review methods in the United States. Int J Qual Health Care. 1995; 7:156-163.

3. Salvador Peiró, Eduard Portella Identificación del uso inapropiado de la hospitalización: la búsqueda de la eficiencia. Med Clin (Barc) 1994; 103:65-71.

4. Muiño A, González VJ, Rodríguez E, Lázaro C, Fernández E. Asistencia en un servicio de urgencia: justificación de las visitas y adecuación de los ingresos. Rev Clin Esp 1988; 182:374-8.

5. Matorras P, de Pablo M, Otero L, Alonso F, Daroca $\mathrm{R}$, Díaz-Caneja N. Adecuación de los ingresos en un servicio de medicina interna de un hospital de tercer nivel. Med Clin (Barc) 1990; 94:290-293.

6. Oterino de la Fuente D, Peiró S, Portella E, Marchan C, Aymerich S. Utilización innecesaria de la hospitalización: importancia de la gestión a nivel de servicio. Rev Calidad Asistencial 1994; 1: 4-8.

7. Lorenzo S. El retorno del AEP. Vigencia y cambios en su utilización. Rev Calidad Asistencial 2002; $17: 587-88$

8. Villalta J, Siso A, Cereijo AC, Sequira E, de la Sierra A. Adecuación de la hospitalización en una unidad de estancia corta de un hospital universitario. Un estudio controlado. Med Clin (Barc) 2004; 122:454-6.

9. Navarro G, Prats-Marín MA, Menacho A, Trilla A, Salleras LL. Review of the utilization of a university hospital in Barcelona (Spain): Evolution 19921996. Eur J Epidemiology 2001; 17:679-84.

10. Oterino de la Fuente D, Peiró S, Marchan C, Porte1la E. Inappropiate Hospitalitation: reasons and determinants. Euro J Public Health 1996; 6:126132.

11. Lorenzo S Revisión de la utilización de recursos: estudios realizados en España. Rev Calidad Asistencial 1997; 12:140-6.

12. Zambrana JL, Delgado M, Cruz G, Diez F, Martín MD, Salas C. Factores asociados a ingresos inadecuados en un servicio de medicina interna. Med Clin (Barc) 2001; 116:652-4.
13. Suárez García F, Oterino de la Fuente D, Peiró S, Librero J, Barrero C, Parras N, Crespo MA, PérezMartín A. Factores asociados al uso y adecuación de la hospitalización en personas mayores de 64 años. Rev Esp Salud Pública 2001; 75:237-248.

14. Antón P, Peiró S, Aranaz JM, Calpena R, Company A. AdeQuos, un instrumento para la evaluación de la adecuación de la hospitalización en el pase de visita. Rev Calidad Asistencial 2002; 17:591-9.

15. Moyá-Ruiz C, Peiró S, Meneu R. Effectiveness of feedback to physicians in reducing inappropriate use of hospitalization. Int J Qual Health Care 2002; 14:305-12.

16. Martínez M. Impacto de una intervención continuada sobre la reducción de la utilización inadecuada de la hospitalización en un servicio de medicina interna. [Tesis doctoral]. Las Palmas: Universidad de las Palmas de Gran Canaria; 2003.

17. Payne SM, Restuccia JD. The role of feddback in reducing medically unnecessary hospital use. Med Care 1991; 29 (suppl) 91-106.

18. Meneu R. ¿AEP para que? Rev Calidad Asistencial 2002; 17:589-590.

19. Peiró S, Meneu R, Roselló ML, Portella E, Carbonell-Sanchís R, Fernández C et al. Validez del protocolo de evaluación del uso inapropiado de la hospitalización. Med Clin (Barc) 1996; 107:124-29.

20. Peiró S Meneu R. Uso inapropiado de la hospitalización: problemas en los diseños muestrales. Gac Sanit 1997; 11:103-104.

21. Rodriguez-Vera F, Alcoucer MR, Rodriguez FJ, Camacho Tcolchero J, Pujol E. Adecuación de los ingresos en un servicio de medicina interna de un hospital de segundo nivel. An Med Interna (Madrid) 1999; 16:277-280.

22. Portella E, Oterino de la Fuente D, Peiro S. Impact de l'agé sur la utilization non necesaire. En: La dependence, defi de l'an 2.000. Geneve: JC Rey C Tilquin. 1994. p.174-179

23. Perneger TV, Chopard P, Sarasin FP, Gaspoz JM, Lovis C, Unger PF, et al. Risk factor for a medically inappropriate admission to a department of internal medicine. Arch Intern Med 1997; 157:1495-500

24. Apolone G, Alfieri V, Braga A et al. A survey of the necessity of the hospitalization day in an Italian Teaching Hospital. Qual Assur Health Care 1991; 3:1-9. 
25. Ollero M. Adecuación y utilidad del ingreso hospitalario. Med Clin (Barc) 2001; 116:655-657.

26. Cordero A, Aguila J, Massalana A, Escoto V, Lopes L, Susano R. Adequaçao dos internamentos nun serviço de medicina. Aplicaçao da escala AEP. Acta Med Port 2004; 17:113-8.

27. Médicos de Familia e internistas trabajando por una atención integral. Sevilla: Sociedad Española de Medicina Interna y Sociedad Española de Medicina Familiar y Comunitaria; 1998.

28. Minué S, De Manuel E, Solas O. Situación actual y futuro de la atención primaria. En: Informe SESPAS 2002. Valencia: SESPAS. P.395-438.
29. Castaño EJ. Variables que influyen en la tasa de derivación de los centros de salud de Mallorca. En: Avances en la gestión sanitaria: implicaciones para la política, las organizaciones sanitarias y la práctica clínica. Palma de Mallorca: Asociación de Economia de la Salud; 2000. p. 315-321.

30. Badià G. ¿El hospital, debe competir dominar o compartir con la atención primaria? Aten Primaria 1998; 21: 186-9.

31. Aranaz J, M Buil JA. Gestión Sanitaria: acerca de la coordinación entre niveles asistenciales. Med Clin (Barc) 1996; 106:182-4. 